\title{
INVESTIGATION OF VOLTAGE BREAKDOWN CAUSED BY MICROPARTICLES*
}

\author{
G.R. Werner, ${ }^{\dagger}$ J.C. Betzwieser, J. Knobloch, H. Padamsee, M. Qureshi, J.E. Shipman, \\ Laboratory of Nuclear Studies, Cornell University, Ithaca, NY 14853, USA \\ P.J. McKeown, Evans East Analytical Services, East Windsor, NJ 08520, USA
}

\begin{abstract}
Degradation of RF accelerating cavities caused by field emission currently limits the design of future linear accelerators. When field emission is the problem, foreign particles often deserve the blame, but they can be "processed" away if the cavity electric field is high enough to initiate voltage breakdown near the particle site, in which case the troublesome particle vaporizes, leaving behind a crater surrounded by a starburst-shaped feature. Severe cratering, however, erodes cavity surfaces to the point of significantly diminished acceleration capability. Breakdown events producing craters and starbursts similar to those seen in RF cavities can also be incited by a DC electric field. After intentionally contaminating niobium and copper cathodes with different kinds of micron-sized particles, we used scanning electron microscopes with EDX and AES capabilities to obtain "before" and "after" pictures of particles that vaporized under DC electric fields between 30 and $150 \mathrm{MV} / \mathrm{m}$.
\end{abstract}

\section{VOLTAGE BREAKDOWN LIMITS CAVITY PERFORMANCE}

When the electric field in an RF accelerator cavity becomes strong enough, breakdown (arcing) of the cavity vacuum may occur. An arc depletes the energy stored in the cavity, drastically lowering the electric field and disabling the accelerator; the cavity field is therefore limited to a safe level below its breakdown field. The breakdown field can be increased by processing - intentionally allowing breakdown in hopes that the arc will destroy whatever initiated the breakdown, preventing future trouble. However, too much arcing erodes the walls of the cavity-especially a normal conducting cavity with large input power-enough to cause serious losses in performance.

\section{RF AND DC BREAKDOWN SIMILAR}

Breakdown of a DC voltage gap in many ways resembles RF breakdown. Most striking, both phenomena leave distinctive starburst patterns on the surface where the breakdown was initiated (figure 1); usually the center of the starburst contains craters.

The relative simplicity of DC apparatus, requiring less exotic paraphernalia than RF cavities, allows faster and cheaper experimentation; we can more efficiently learn about RF breakdown using the results of DC experiments

\footnotetext{
* Work supported by NSF

† grw9@cornell.edu
}

to guide RF research. For example, we may study the effect of different cathode-surface treatments on the breakdown voltage of a vacuum gap, and then test only the most successful treatments on RF cavities.

\section{DC BREAKDOWN}

\subsection{Contaminant Particles}

Surface particles are in many cases responsible for unexpectedly high field emission and low breakdown fields, both in DC gaps and RF cavities. Examination of superconducting niobium cavities with a scanning electron microscope (SEM) and Auger electron spectroscopy (AES) [1] has revealed contaminants (e.g., In, $\mathrm{Fe}, \mathrm{Cr}, \mathrm{Si}, \mathrm{Cu}$ ) at field emission and breakdown sites. It is now accepted that superconducting cavities must be etched cleanly and assembled in particle-free environments to avoid limitation to low fields.

By intentionally contaminating the cathode of a high voltage vacuum gap with particles, mapping the locations of all particles with the SEM, applying a high field (30$150 \mathrm{MV} / \mathrm{m}$ ), and examining changes in the SEM, we have shown that if contaminant particles are present on a niobium cathode, breakdown occurs almost always (and possibly without exception) at particle sites. Figure 2 shows typical examples. Copper cathodes show the same propensity to break down at particle sites, but we do not yet have enough data to draw as certain a conclusion.

Efforts to determine what properties of particles lead to lower breakdown fields have not yet proved conclusive, with the exception of milled vanadium particlesextremely spiny particles usually 5-20 microns in size (see figure 3), which field-emit and cause breakdown at lower fields than other kinds of particles we've tried (both on $\mathrm{Cu}$ and $\mathrm{Nb}$ cathodes). Such spiky particles suggest that geometric field enhancement (leading to high field emission current densities) contributes to the relatively low breakdown fields.

\subsection{Starbursts and Craters}

Starbursts (see figure 1) marking the site of breakdown in DC gaps and RF cavities are so similarly distinctive as to establish a close connection between RF and DC breakdown. Starbursts usually show up as a star-shaped areas darker than the surroundings in a secondary emission SEM image; often a starburst contains several craters.

Auger analysis, which is sensitive to the first $10 \mathrm{~nm}$ surface layer, shows that starbursts are "cleaner" than sur- 


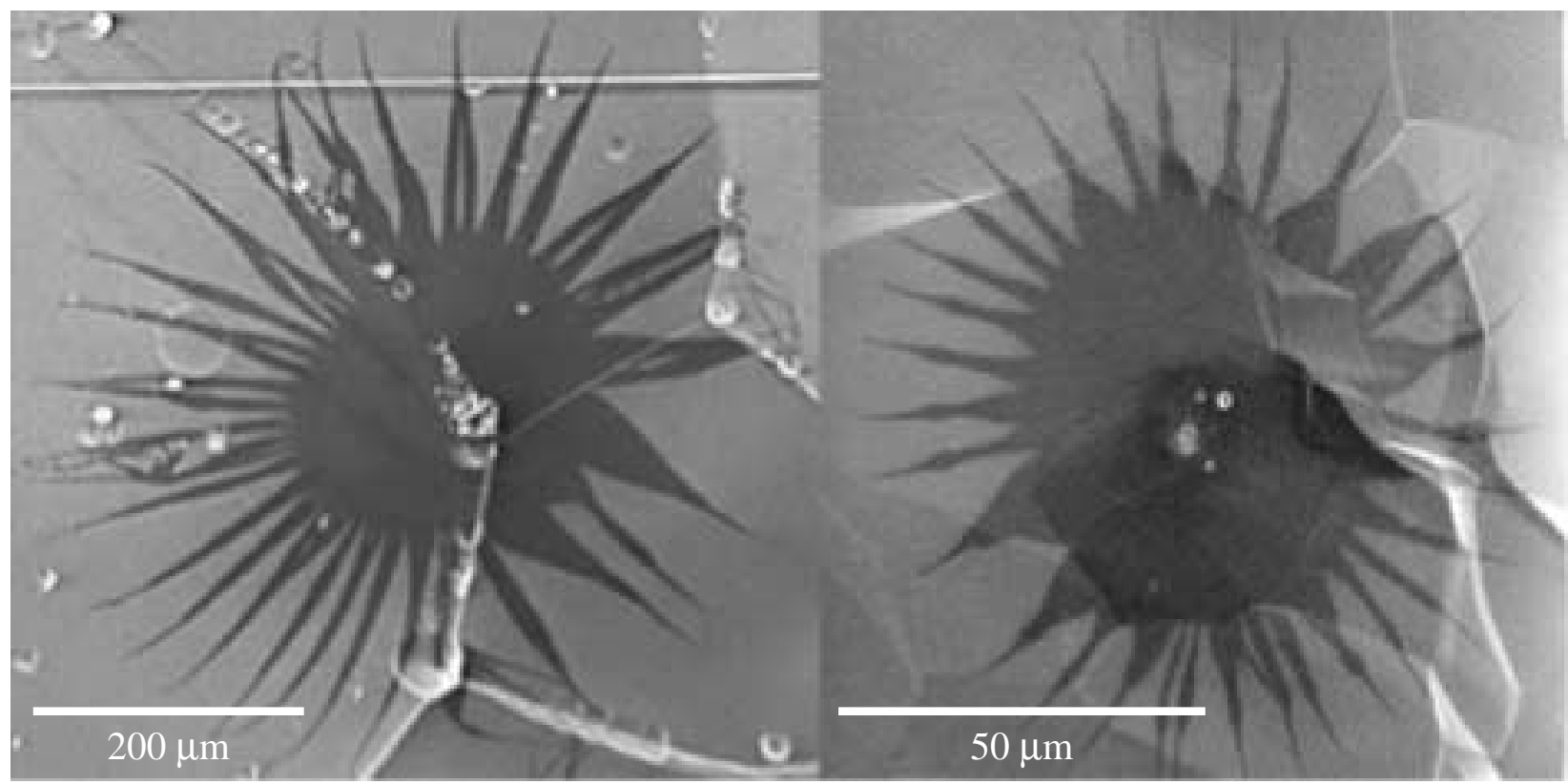

Figure 1: RF and DC starbursts: from $1.5 \mathrm{GHz}$ superconducting cavity (left), and room temperature DC voltage gap (right). Note that this RF starburst is actually about four times larger than than the pictured DC starburst.

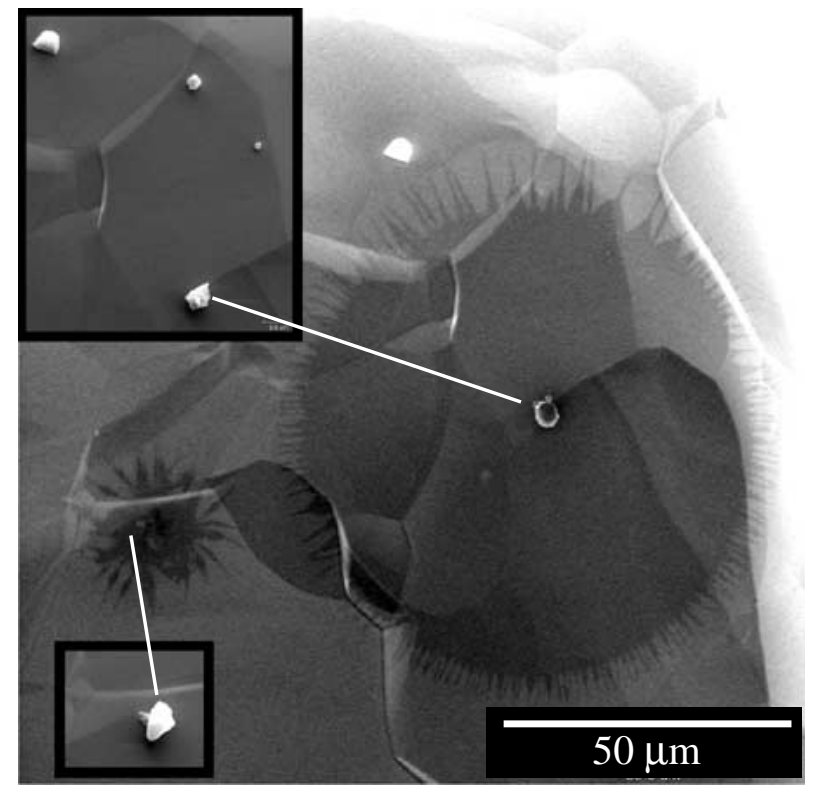

Figure 2: Two starbursts that occurred at 50MV/m (gap distance $225 \mu \mathrm{m}$ ); the two insets show the particles before the breakdown (lower inset became left starburst, upper inset became right starburst).

rounding areas. We knew that starbursts on niobium lack fluorine [1] (otherwise present in the surface due to etching in hydrofluoric acid), but only recently found that they contain less carbon (from carbon dioxide and monoxide, and hydrocarbons) than the surfaces untouched by the breakdown event (figure 4). Considering the cleaning effect of ion bombardment during a glow discharge, we believe that

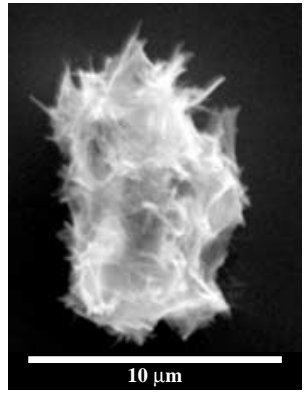

Figure 3: A typical vanadium particle; this particle was at the center of the (DC) starburst shown in figure 1 .

the starburst outlines the extent of intense plasma activity. Also on copper cathodes we see starbursts, which, though sometimes dimly defined in SEM images, are clearly illuminated by AES techniques. Starbursts on copper appear to be on the average much more scraggly than niobium starbursts.

Starbursts with central craters are very common on niobium, but starbursts on an electropolished copper plate feature small craters scattered uniformly throughout the carbon-depleted region (figure 5). Two obvious differences between copper and niobium that could affect the starburst and the amount of cratering are: (1) the melting temperature of copper is much lower than of niobium, and (2) the oxide layer of copper is much thinner than that of niobium.

Niobium naturally accumulates a relatively thick oxide (3-5nm), which by means of anodization can grow much thicker. Our first test on a niobium cathode with a $20 \mathrm{~nm}$ thick oxide layer produced unusual "starbursts," lacking 


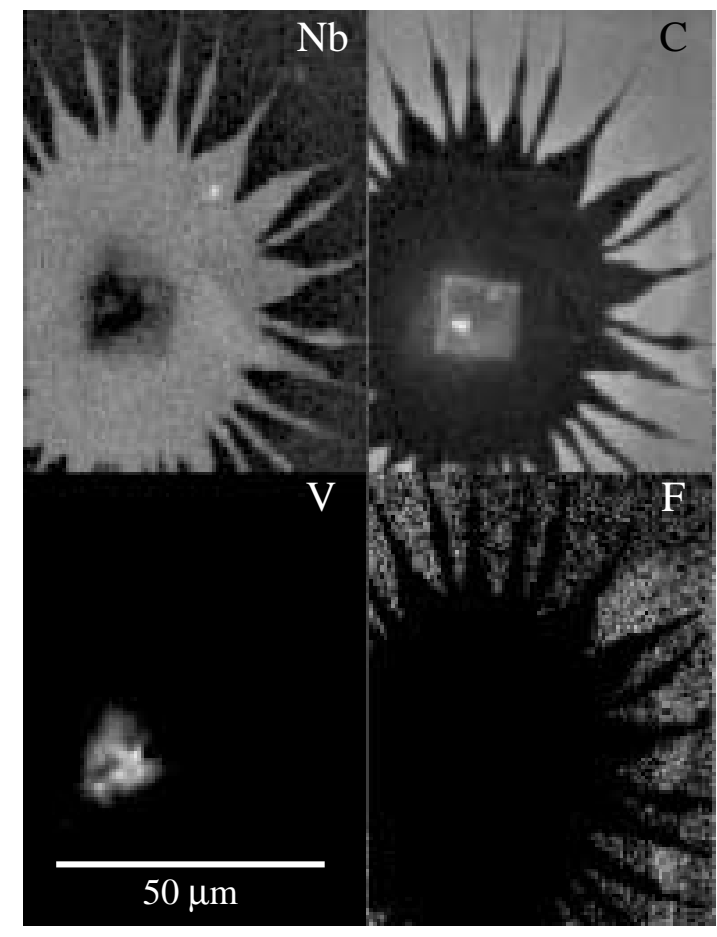

Figure 4: The abundance of the elements $\mathrm{Nb}, \mathrm{C}, \mathrm{V}$, and $\mathrm{F}$ (in a $10 \mathrm{~nm}$ thick surface layer) in the starburst shown in figure 1. Lighter areas contain lower concentrations. Originally there was a $\mathrm{V}$ particle at the center (see figure 3); the breakdown event cleaned away all $\mathrm{F}$ and some $\mathrm{C}$, providing the contrast to see the starburst in the SEM image.

craters entirely as well as the contrast in SEM images (we have not yet seen AES images), as in figure 6. Almost all featured a molten center.

\section{CONCLUSIONS}

1. RF and DC breakdown processes have much in common.

2. With contaminant particles present on the cathode, breakdown is highly likely to occur at a particle site; for DC breakdown this has been directly verified in "before" and "after" pictures.

3. Spiky vanadium particles are excellent field emitters and cause breakdown at relatively low electric fields.

4. The starburst patterns are areas that have been "cleaned" of surface contaminants, notably carbon, by plasma activity.

5. Even though a contaminant particle is primarily responsible for a breakdown event, the nature of the resulting starburst depends on the substrate cathode material.

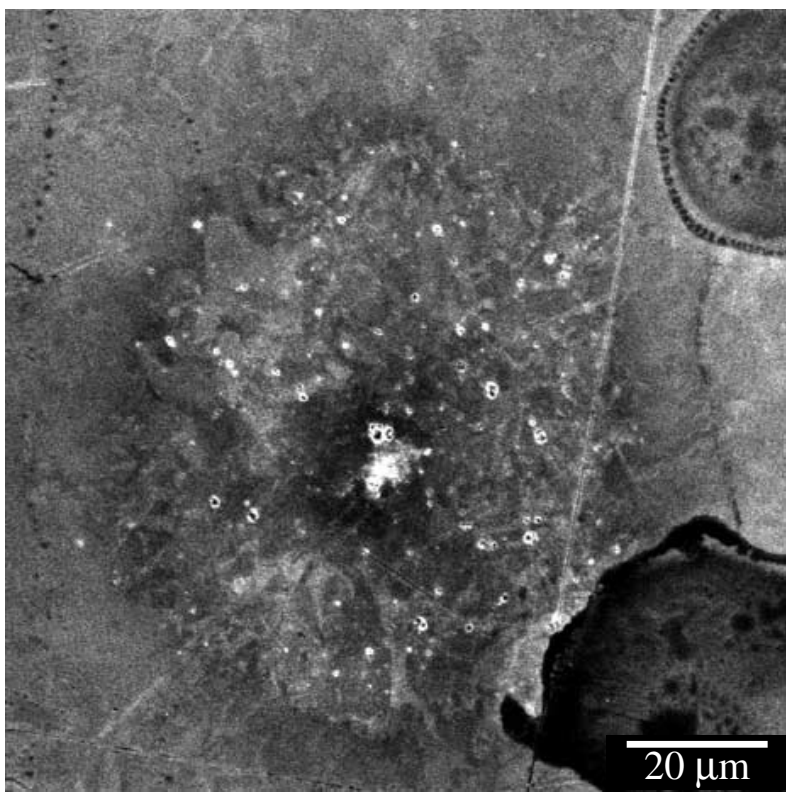

Figure 5: A starburst on electropolished copper.

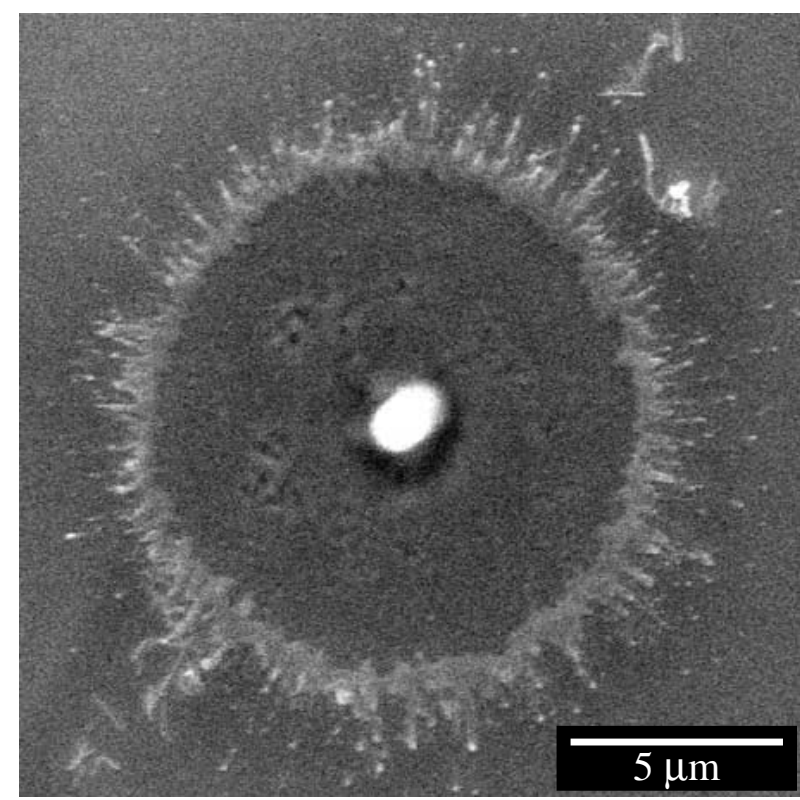

Figure 6: A starburst on niobium with a 20nm thick oxide.

\section{REFERENCES}

[1] T. Hays, M. Klauda, J. Knobloch, D. Moffat, and H. Padamsee, "Microscopic Examination and Elemental Analysis of Field Emission Sites in $5.8 \mathrm{GHz}$ Superconducting Mushroom Cavities," Proceedings of the 6th Workshop on RF Superconductivity, CEBAF, Newport News, VA, USA. 